\title{
Tune calculation at the Injection
}

\author{
Xi Yang
}

March 28, 2007

\begin{abstract}
Injection tunes in the Booster can be adjusted via quadrupole correctors. Quadrupole correctors at short and long sections are ramped as two different groups, and they control horizontal and vertical tunes separately since the horizontal beta function reaches the maximum at short sections and the vertical beta function reaches the maximum at long sections. A simple linear model is used to calculate the tune based upon quad corrector settings.
\end{abstract}

\section{Method}

Quads at short and long sections are represented by $Q S$ and $Q L$, and their currents are $I_{\text {tunes }}$ and $I_{\text {tunel. }}$. Horizontal and vertical tunes are $v_{x}$ and $v_{y}$. Equations (1) and (2) are used to calculate $v_{x}$ and $v_{y}$ once $a, b, c, d, e$, and $f$ are known.

$$
\begin{aligned}
& v_{x}=a \cdot I_{\text {tunes }}+b \cdot I_{\text {tunel }}+e \\
& v_{y}=c \cdot I_{\text {tunes }}+d \cdot I_{\text {tunel }}+f
\end{aligned}
$$

Coefficients $a, b, c, d, e$, and $f$ are to be determined, and first is to find $a$ and $c$. $I_{\text {tunel }}$ is fixed at $0.54 \mathrm{~A}$, and $I_{\text {tunes }}$ is varied from $-0.35 \mathrm{~A}$ to $0.55 \mathrm{~A}$ with a step of $0.1 \mathrm{~A} ; v_{x}$ and $v_{y}$ are calculated at each set of $I_{\text {tunes }}$ and $I_{\text {tunel }}$ using MAD. Afterwards, equations (3) and (4) are used to calculate $a$ and $c$, and results are " $a=0.43735$ " and " $c=-0.07385$ ".

$$
\begin{array}{ll}
\overline{\Delta v_{x}}=a \cdot \Delta I_{\text {tunes }} & \text { (3) } \\
\overline{\Delta v_{y}}=c \cdot \Delta I_{\text {tunes }} & \\
\Delta I_{\text {tunes }}=0.1 \mathrm{~A}, & \overline{\Delta v_{x}}=\sum_{i=1}^{9}\left(\Delta v_{x}\right)_{i} / 9, \quad \overline{\Delta v_{y}}=\sum_{i=1}^{9}\left(\Delta v_{y}\right)_{i} / 9
\end{array}
$$

Second, $b$ and $d$ are to be determined. $I_{\text {tunes }}$ is fixed at $-0.2178 \mathrm{~A}$, and $I_{\text {tunel }}$ is varied from $0.04 \mathrm{~A}$ to $0.54 \mathrm{~A}$ with a step of $0.1 \mathrm{~A} ; v_{x}$ and $v_{y}$ are calculated at each set of $I_{\text {tunes }}$ and $I_{\text {tunel }}$ using MAD. Afterwards, equations (5) and (6) are used to calculate $b$ and $d$, and results are " $b=0.08529 "$ and " $d=-0.2401 "$.

$$
\begin{aligned}
& \overline{\Delta v_{x}}=b \cdot \Delta I_{\text {tunel }} \\
& \overline{\Delta v_{y}}=d \cdot \Delta I_{\text {tunel }} \\
& \Delta I_{\text {tunel }}=0.1 \mathrm{~A}, \quad \overline{\Delta v_{x}}=\sum_{i=1}^{5}\left(\Delta v_{x}\right)_{i} / 5, \quad \overline{\Delta v_{y}}=\sum_{i=1}^{5}\left(\Delta v_{y}\right)_{i} / 5
\end{aligned}
$$


Finally, $e$ and $f$ are determined using equations (1) and (2) since $a, b, c$, and $d$ are already known, and results are " $e=6.70475$ " and " $f=6.83989 "$ ".

We can calculate the lattice tune at injection using equation (7) and (8) with the input of quad corrector settings, $I_{\text {tunes }}$ and $I_{\text {tunel }}$.

$$
\begin{aligned}
& v_{x}=0.43735 \cdot I_{\text {tunes }}+0.08529 \cdot I_{\text {tunel }}+6.70475 \\
& v_{y}=-0.07385 \cdot I_{\text {tunes }}-0.2401 \cdot I_{\text {tunel }}+6.8400
\end{aligned}
$$

\section{Check the result}

The tune shift due to quad correctors can be estimated using equation (9).[1]

$$
\delta v_{x, y}=\frac{1}{4 \pi} \sum_{\mathrm{i}} \frac{\left(\beta_{x, y}\right)_{\mathrm{i}}}{f_{i}}
$$

When " $I_{\text {tunel }}=0.0 \mathrm{~A}$ " and " $I_{\text {tunes }}=0.0 \mathrm{~A}$ ", " $v_{x}=6.704553$ " is obtained using MAD. " $\beta_{\mathrm{x}}$ $\approx 33.0 \mathrm{~m}$ " at short sections, " $\beta_{\mathrm{x}} \approx 7.0 \mathrm{~m}$ " at long sections, and $f^{-1}$ can be calculated using equation (10).[2]

$$
\begin{aligned}
& 1 / \mathrm{f}=0.3 \cdot k_{\text {quad }} \cdot I_{\text {tune }} \cdot l /\left(P_{c} \cdot l\right) \\
& k_{\text {quad }}=0.02178 \mathrm{~T} / \mathrm{m}, P_{c}=0.954263 \mathrm{GeV} / \mathrm{c} \text { at injection }
\end{aligned}
$$

Here, $f$ can be $f_{s}$ or $f_{l}$, and $I_{\text {tune }}$ can be $I_{\text {tunes }}$ or $I_{\text {tunel }}$.

When " $I_{\text {tunel }}=0.54 \mathrm{~A}$ " and " $I_{\text {tunes }}=0.35 \mathrm{~A}$ ", $\delta v_{x}$ is about 0.151 from quads at short sections, and it's about 0.05 from quads at long sections, so $v_{x}$ is estimated to be $6.9055(=6.70455+0.151+0.05)$ using equation (9).

Using the linear model, we get $v_{x}=0.43735 \cdot 0.35+0.08529 \cdot 0.54+6.70475=6.9039$, and it's very close to the result which is estimated using equation (9).

\section{Conclusion}

The injection tune calculated by the linear model has been checked using the analytical formula, and there is a good agreement. The linear model for the tune calculation can be applied to the operation or the tune scan at injection.

\section{Acknowledgement}

Francois Ostiguy for useful discussions.

\section{References:}

[1] D. A. Edwards and M. J. Syphers, An Introduction to the Physics of High Energy Accelerators.

[2] A. Drozhdin, Booster MAD input file. 